\title{
A Novel Maize MicroRNA Negatively Regulates the Resistance to Fusarium Verticillioides
}

\author{
Yufang $\mathrm{Xu}^{1}$, Renjie Wang ${ }^{1}$, Peipei $\mathrm{Ma}^{1}$, Yan $\mathrm{Cao}^{1}$, Tao $\mathrm{Li}^{1}$, Jianyu $\mathrm{Wu}^{1}$, and Huiyong \\ Zhang $^{1}$ \\ ${ }^{1}$ Henan Agricultural University
}

January 20, 2022

\begin{abstract}
Despite miRNAs regulate the defense response against multiple pathogenic fungi in diverse plant species, few efforts had been devoted to deciphering the involvement of miRNA in resistance to Fusarium verticillioides ( F. verticillioides), a major pathogenic fungal in maize production. In this study, we discovered a novel $F$. verticillioides-responsive miRNA designated zma-unmiR4 in maize kernels. The expression levels of zma-unmiR4 were significantly repressed in the resistant maize line but induced in the susceptible lines upon $F$. verticillioides exposure, whereas its target gene ZmGA2ox4 exhibited the opposite pattern of expression. Heterologous overexpression of zma-unmiR4 in Arabidopsis resulted in enhanced growth and compromised resistance to $F$. verticillioides. By contrast, transgenic plants overexpressing ZmGA2ox4 or the homolog AtGA2ox 7 showed impaired growth and enhanced resistance to F. verticillioides. Moreover, zma-unmiR4-mediated suppression of AtGA2ox7 disturbed the accumulation of bioactive gibberellin (GA) in transgenic plants and perturbed a set of defense-related genes in response to $F$. verticillioides. Exogenous application of GA or GA biosynthetic inhibitor could modulate F. verticillioides resistance in different plants. Taken together, our results suggest that zma-unmiR4- ZmGA2ox4 module might act as a major player in balancing growth and the resistance to $F$. verticillioides pathogen in maize.
\end{abstract}

Title: A Novel Maize MicroRNA Negatively Regulates the Resistance to Fusarium Verticillioides

Running title: zma-unmiR4 confers Fusarium Verticillioides susceptibility

Authors: Yufang $\mathrm{Xu}^{1 \#}$, Renjie Wang ${ }^{1 \#}$, Peipei $\mathrm{Ma}^{1}$, Yan $\mathrm{Cao}^{1}$, Tao $\mathrm{Li}^{1}$, Jianyu $\mathrm{Wu}^{1,2}$, Huiyong Zhang ${ }^{1,2^{*}}$

${ }^{1}$ College of Life Sciences, Henan Agricultural University, Zhengzhou 450002, China

${ }^{2}$ State Key Laboratory of Wheat and Maize Crop Science, Collaborative Innovation Center of Henan Grain Crops, Henan Agricultural University, Zhengzhou 450002, China

\# These authors contributed equally to this work.

* Corresponding author: Huiyong Zhang (huiyong.zhang@henau.edu.cn)

Funding: This work was supported by the Joint Funds of the National Natural Science Foundation of China (Grant No. U2004157 to H.Z), the International (Regional) Joint Research Project of the National Natural Science Foundation of China (Grant No. 31761143009 to J.W), and the Innovation Special Program of Henan Agricultural University for Science and Technology (Grant No. 30501044 to Y.X.).

\begin{abstract}
Despite miRNAs regulate the defense response against multiple pathogenic fungi in diverse plant species, few efforts had been devoted to deciphering the involvement of miRNA in resistance to Fusarium verticillioides (F. verticillioides ), a major pathogenic fungal in maize production. In this study, we discovered a novel
\end{abstract}


F. verticillioides- responsive miRNA designated zma-unmiR4 in maize kernels. The expression levels of zma-unmiR4 were significantly repressed in the resistant maize line but induced in the susceptible lines upon F. verticillioides exposure, whereas its target geneZmGA2ox4 exhibited the opposite pattern of expression. Heterologous overexpression of zma-unmiR4 in Arabidopsisresulted in enhanced growth and compromised resistance to $F$. verticillioides . By contrast, transgenic plants overexpressing ZmGA20x4 or the homolog AtGA20x7 showed impaired growth and enhanced resistance to $F$. verticillioides . Moreover, zma-unmiR4mediated suppression of AtGA2oxy disturbed the accumulation of bioactive gibberellin (GA) in transgenic plants and perturbed a set of defense-related genes in response to $F$. verticillioides . Exogenous application of GA or GA biosynthetic inhibitor could modulate $F$. verticillioides resistance in different plants. Taken together, our results suggest that zma-unmiR4- ZmGA20x4 module might act as a major player in balancing growth and the resistance to $F$. verticillioidespathogen in maize.

Key words : microRNA, zma-unmiR4, Fusarium Verticillioides, Disease resistance, Gibberellin

\section{Summary statement}

Characterization of the roles of miRNA in Fusarium verticillioides resistance have a profound impact for controlling the pathogene damages. By functional analysis of the novel $F$. verticillioides- responsive miRNA zma-unmiR4, we demonstrate that zma-unmiR4-ZmGA20x4/AtGA2ox7 mediated bioactive GA dynamics played as a crucial regulator in $F$. verticillioides resistance and plant growth.

\section{INTRODUCTION}

MicroRNAs (miRNAs), a class of 20-24 nucleotides endogenous non-coding RNAs, have been widely found in eukaryotes and play as gene repressors by directing the cleavage or translational repression of the target transcripts (Voinnet, 2009). It has been well documented that miRNAs are involved in the control of some of the most challenging plant traits in agricultural production, such as plant development and architecture, and environmental stress and defense responses (Kumar, 2014; Rubio-Somoza and Weigel, 2011; Sunkar et al., 2012).

Accumulating evidences indicate that miRNAs regulate multiple biotic stress responses in plants, including the interactions with fungi (Chen et al., 2021; Hu et al., 2020; Zhang et al., 2016), viruses (Mengistu and Tenkegna, 2021; Yang et al., 2016; Yao et al., 2019), bacteria (Liu et al., 2019; Navarro et al., 2006; Zhang et al., 2011), as well as insects (Feng et al., 2021; Li et al., 2018). miR393, the first miRNA identified to be involved in plant immunity, could be induced by bacterial flagellin-derived peptide and contributes to restrict the growth of Pseudomonas syringae by repressing auxin signaling (Navarro et al., 2006). miR159a was indicated to play a positive role in rice resistance to Magnaporthe oryzae(Chen et al., 2021), while miR156 negatively regulates rice resistance to bacterial blight by Xanthomonas oryzae (Liu et al., 2019). In addition, the important roles of miR160a, miR166, miR528, miR398b, miR164, and miR168 in disease resistance had been well characterized by regulating specific target genes in various crops (Li et al., 2014). For instance, miR528 negatively regulated rice resistance to stripe virus by cleaving the transcripts of $L$ ascorbate oxidase $(A O)$ gene (Wu et al., 2017). Loss function of the Osa-miR159a target genes, including $O s G A M Y B$, OsGAMYBL, and OsZF, resulted in enhanced resistance to $M$. oryzae , consistent with the related phenotypes of Osa-miR159a overaccumulation plants (Chen et al., 2021). miR156 negatively regulated rice resistance against bacterial blight though decreasing its targets IPA1 and OsSPL7 expression levels (Liu et al., 2019). Considering the extensive regulation of miRNAs during plant immunity, further characterization of pathogen-responsive miRNAs and resultant miRNA-mediated disease defense processes will have a profound impact on the development of new strategies for controlling disease damages in crop production.

Fusarium verticillioides (F. verticillioides) is one of the most common pathogenic fungus that causes various prevalent diseases in crops, especially for maize, posing a great challenge to food and feed safety (Gai et al., 2018; Ju et al., 2017; Liu et al., 2020; Mu et al., 2018; Septiani et al., 2019). F. verticillioides infection occurs throughout the whole growth period of maize and results in seedling blight, stalk rot, ear rot, and seed rot (Machado et al., 2013; Septiani et al., 2019; Stagnati et al., 2019). Most importantly,F. verticillioides 
-infected plants or seeds may accumulate fumonisins, a family of mycotoxins associated with several diseases in livestock and humans and are classified as probable carcinogens (Rosa Junior et al., 2019). Thus, it is of great significance to dissect the molecular mechanism of resistance to $F$. verticillioides . Although many genetic studies and omics data have identified a series of QTLs/genes associated with $F$. verticillioides resistance (Butrón et al., 2019; Chen et al., 2016; Lanubile et al., 2017; Maschietto et al., 2017; Schiwek et al., 2020; Yao et al., 2020), the molecular mechanism of plant against to $F$. verticillioides remain largely elusive, especially the role of miRNAs in this process. In our previous study (Zhou et al., 2020), a number of miRNAs including known and new predicted miRNAs were identified to be potentially associated with the resistance to $F$. verticillioides ear rot via high-throughput sequencing. Further functional analysis of these miRNAs becomes important to dissect the molecular mechanism of plant- $F$. verticillioides interaction and ultimately disease resistance improvement.

In current study, we focused on a novel $F$. verticillioides- responsive miRNA designated zma-unmiR4, and aimed to reveal its function in plants against $F$. verticillioides. We found that the expression levels of zma-unmiR4 was significantly downregulated in the resistant maize line but upregulated in the susceptible lines after $F$. verticillioides infection, whereas the target gene ZmGA20x4 displayed the opposite profiles of expression. Heterologous accumulation of zma-unmiR4 resulted in impaired resistance to $F$. verticillioides infection and enhanced growth inArabidopsis, however, the transgenic plants overexpressing ZmGA2ox4 or the homolog AtGA20x7 showed high resistance toF. verticillioides as well as retarded growth. Further analyses indicated that zma-unmiR4 was able to regulate $F$. verticillioidesresistance through gibberellin (GA) signaling by suppressing AtGA2ox 7 expression inArabidopsis . These results provide direct evidence for the crucial role of zma-unmiR4 in regulating plant growth and disease resistance to $F$. verticillioides .

\section{MATERIALS AND METHODS}

\section{Plant materials and growth conditions}

The $F$. verticillioides -susceptible maize inbred line N6 is a Tangsipingtou lines, while the $F$. verticillioides -resistant maize line BT-1 is improved by tropical Asia material (Wang et al., 2016). Healthy maize seeds were selected and sowed, and seedlings were grown in a climate-controlled culture room at $24 \pm 2{ }^{\circ} \mathrm{C}$ with a 14/10-h light/dark photoperiod.

Arabidopsis (Columbia) seeds were first sterilized with $75 \%$ ethyl alcohol for 60 s, and then soaked in $3 \%$ sodium hypochlorite for $10 \mathrm{~min}$. Surface-sterilized seeds were stratified in the dark at $4^{\circ} \mathrm{C}$ for 2 days and sowed on MS dishes ( $\mathrm{pH}$ 5.7) for 7 days, then seedlings were transferred to sterilized nutritional soil at 22 ${ }^{\circ} \mathrm{C}$ with a 16/8-h light/dark photoperiod.

The healthy seeds of japonica rice KY131 were soaked in 3\% sodium hypochlorite for 30 min, and were accelerated germinated at $37^{\circ} \mathrm{C}$ for 3 days. Then the germinating seeds were sowed into 96 -well plates, and water-cultured at $28 \pm 2{ }^{\circ} \mathrm{C}$ with a $14 / 10$-h light/dark photoperiod.

\section{Vector construction and generation of transgenicArabidopsis plants}

For zma-unmiR 4 overexpression (zma-unmiR 4 OE ), the 398-bp hairpin region of zma-unmiR4 was amplified from genomic DNA of N6 and ligated into the binary vector pJim19 (Bar) driven by the 35S promoter. For ZmGA2ox4 overexpression ( $Z m G A 20 x 4 O E$ ) and AtGA2oxy overexpression (AtGA2oxy OE ), the coding sequences of $Z m G A 20 x 4$ and AtGA2oxy from ATG to TGA was amplified and ligated into the binary vector pCANBIA1302 (HYG), respectively, driven by the 35S promoter. After confirming the sequence,zma-unmiR 4 $O E, Z m G A 20 x 4 O E$ and AtGA20x7OE vectors were introduced into Agrobacterium tumefaciensstrain GV3101 and transferred into Col-0 by floral dip. For 35S::ZmGA2ox4-GUS and 35S::AtGA2ox7-GUS vectors, the coding sequences of $Z m G A 20 x 4$ and AtGA2oxy without stop codon were amplified, respectively, and ligated into the binary vector pCAMBIA1391 (HYG) driven by the 35S promoter. For 35S:: ZmGA2ox4$Y F P$ vector, the coding sequences of ZmGA2ox4 without stop codon were amplified and ligated into the binary vector pGRDR driven by the $35 \mathrm{~S}$ promoter. For the transient expression of zma-unmiR4 in maize protoplasts, the 398-bp hairpin region of zma-unmiR 4 was amplified from genomic DNA of N6 and ligated into 
pPROTO driven by the $35 \mathrm{~S}$ promoter. The primers used for vector construction are listed in Supplementary Table 1.

\section{Transfection ofmaize protoplasts}

Maize protoplasts were isolated from 10-days etiolated seedlings (B73) as previously described (Li et al., 2021). The 35S:: ZmGA2ox4-YFP was co-transfected into the protoplasts with $35 S::$ pre-zma-unmiR4and empty vectors, respectively, using the polyethyleneglycol (PEG)-calcium-mediated transfection. After incubation in dark for $16 \mathrm{~h}$, YFP and mCherry signals were observed using laser scanning confocal microscope (Nikon, A1HD25). The relative fluorescence intensity (YFP/mCherry) was calculated by Image $\mathrm{J}$ software.

\section{F. verticillioides inoculation and phenotype investigation}

TheFusarium verticillioides strain was isolated from naturally infected maize kernels in Zhengzhou. A singlespore of $F$. verticillioides was isolated and propagated on sterilized maize kernels at $28 \mathrm{degC}$ for 7 days. The spores were then collected and diluted to the concentration of $5 \times 10^{6}$ spores $\mathrm{mL}^{-1}$ using sterile distilled water with $0.2 \mu \mathrm{L} / \mathrm{mL}$ Tween 80. The ear inoculation was performed as previously described (Wu et al., 2020; Zhou et al., 2020). The middle of the ears was injected with $2 \mathrm{~mL} \mathrm{~F}$. verticillioides spore suspension $(5 \times$ $10^{6}$ spores $/ \mathrm{mL}$ ) on the fifteenth day after pollination using a syringe. The kernels surrounding the inoculated points were sampled on 0 day and 3 days after inoculation for RNA extraction.

For Arabidopsis leaf inoculation, the healthy rosette leaves of four-weeks plants were inoculated with $20 \mu \mathrm{L}$ $F$. verticillioides spore suspension with the concentration of $1 \times 10^{7}$ spores $/ \mathrm{mL}$, and the control group was inoculated with sterile water. After $4-6$ days culture at $22^{\circ} \mathrm{C}$, the leaf was photographed and sampled for histological staining and $F$. verticillioides quantification. For spore suspension spraying, five-weeks plants were sprayed with F. verticillioides spore suspension $\left(2 \times 10^{7}\right.$ spores $\left./ \mathrm{mL}\right)$ or sterile water once a day for 10 days, the then the plants were photographed. For Arabidopsis seeds inoculation, the sterilized seeds were soaked in F. verticillioides spore suspension with the concentration of $1 \times 10^{7}$ spores $/ \mathrm{mL}$ at $28^{\circ}$ darkness for $48 \mathrm{~h}$, and then taken out and washed with sterile water. The control group was soaked in sterile water. Then the seeds were evenly placed on a wet filter paper in petri dishes at $28^{\circ}$ darkness for 6 days. The disease grades of the seeds rot were investigated according to the criterion showed in Figure S5.

For maize leaf inoculation, the healthy second leaves were cut off and lacerated with a needle, then the 0.3 -cm scratch were injected with $10 \mu \mathrm{L}$ Fusarium verticillioides spore suspension with the concentration of $1 \times 10^{7}$ spores $/ \mathrm{mL}$. After incubation at $25^{\circ} \mathrm{C}$ for $2-5$ days, the leaves were photographed or sampled for $\mathrm{DAB}$ and TB staining.

For rice leaf inoculation, the healthy second leaves were cut off and scratched with a needle, then the leaves were immersed in the $3 \mathrm{mLFusarium}$ verticillioides spore suspension with the concentration of $2 \times 10^{7}$ spores $/ \mathrm{mL}$ and incubated at $25^{\circ} \mathrm{C}$ for 5 days. For rice seedling inoculation, healthy seedlings were sprayed withFusarium verticillioides spore suspension with the concentration of $2 \times 10^{7}$ spores $/ \mathrm{mL}$ once a day for 6 days. Then the leaves were photographed.

\section{RNA analyses}

For protein-coding genes, about $1 \mu \mathrm{g}$ RNA was treated with DNase I (Promega) and reversely transcribed using the Transcriptor First Strand cDNA Synthesis Kit (TOYOBO). The qRT-PCR assay was performed using SYBR Green I Master reagent and a STEP ONE PLUS system (ThermoFisher). The expression levels of target genes were normalized to the expression levels of internal control genes using the $2^{-\Delta \Delta^{\wedge} \tau}$ method. For quantification of protein-coding genes and primary zma-unmiR4 in Arabidopsis, Actin 2 was used as the internal control. For quantification of primary zma-unmiR4 and ZmGA2ox4 in maize, the elongation factor 1 alpha $(E F 1 a)$ of maize was used as the internal control. RNA gel blot analyses of miRNA were performed as described previously (Zhang and Li, 2013). Primer and probe sequences are listed in Supplementary Table 1.

\section{GUS, DAB and TB staining}


Transient expression assay for GUS analysis was performed as previously described (Li et al., 2021). Briefly, 5-weeks Nicotiana benthamiana leaves were infiltrated using Agrobacterium tumefaciens strains GV3101 carrying constructs together with p19 strain. After two days, the leaves were soaked in ice-cold $90 \%$ acetone under vacuum condition for 10min, then washed twice with $100 \mathrm{mM}$ sodium phosphate buffer (pH 7.0) and submerged in dye solution at 37 for $12 \mathrm{~h}$. Chlorophyll was removed by immersing in $95 \%$ ethanol at $95^{\circ} \mathrm{C}$, then samples were photographed by a microscope (Moticam2506).

To visualize $\mathrm{H}_{2} \mathrm{O}_{2}$ accumulation, leaves were immersed in a $0.1 \%$ DAB solution in the Tris- $\mathrm{HCl}$ buffer (pH 6.5) and infiltrated under vacuum condition for $15 \mathrm{~min}$, and then incubated at room temperature for $12 \mathrm{~h}$ in the dark. Then the samples were transferred into the fix solution (60\% ethanol, $20 \%$ acetic acid, $20 \%$ glycerol) at $95^{\circ} \mathrm{C}$ for $5 \mathrm{~min}$. Chlorophyll was removed by immersing in $95 \%$ ethanol at $100^{\circ} \mathrm{C}$, then samples were photographed.

For cell membrane damage visualization, trypan blue staining was performed by submerging the leaves in TB solution $(10 \mathrm{~mL}$ lactic acid, $10 \mathrm{~mL}$ phenol, $10 \mathrm{~mL}$ glycerol, $10 \mathrm{~mL}$ sterile water, and $10 \mathrm{mg}$ trypan blue) for 30-60 min. Chlorophyll was removed by immersing in $95 \%$ ethanol at $100^{\circ} \mathrm{C}$, then samples were photographed.

\section{Hormone treatments}

For maize seedlings, 7-days seedlings were sprayed with $20 \mu \mathrm{M}$ uniconazole, $50 \mu \mathrm{M}$ GA3, or water as the control once a day for 7 days, then the seedlings were photographed and the leaves were inoculated. For rice seedlings, 14-days seedlings were sprayed with $20 \mu \mathrm{M}$ uniconazole, $50 \mu \mathrm{M}$ GA3, or water once a day for 4 days, then the seedlings were sprayed with F. verticillioides spore suspension. For Arabidopsis plants, 17-days seedlings were sprayed with $20 \mu \mathrm{M}$ uniconazole, $50 \mu \mathrm{M}$ GA3, or water once a day for 5 days. Then the seedlings were photographed and leaves were inoculated as the methods described above.

\section{Determination ofchlorophyll concentration}

The measurement of chlorophyll concentration was performed as previously described (Arnon, 1949). Briefly, $0.2 \mathrm{~g}$ leaves from 5-weeks plants were sampled, well ground, and resuspended using $10 \mathrm{~mL} 80 \%$ acetone. The supernatant was then transferred to a new tube after centrifuged at $4000 \mathrm{rpm}$ for $5 \mathrm{~min}$ and then the absorbance was measured at $663 \mathrm{~nm}, 645 \mathrm{~nm}$ and $652 \mathrm{~nm}$, respectively, using an ELISA instrument. The chlorophyll concentration was calculated according the method as previously described (Arnon, 1949).

\section{Gibberellin measurement}

The healthy rosette leaves of four-weeks plants were harvested, immediately frozen in liquid nitrogen, and ground into powder. $50 \mathrm{mg}$ of plant sample was weighed and dissolved in $500 \mu \mathrm{L}$ HPLC-grade acetonitrile $/ \mathrm{H}_{2} \mathrm{O}(90: 10, \mathrm{v} / \mathrm{v}) . \quad 10 \mu \mathrm{L}$ internal standard mixed solution $(100 \mathrm{ng} / \mathrm{mL})$ was added into the extract as internal standards (IS) for the quantication. GAs contents were detected by MetWare (http://www.metware.cn/) based on the AB Sciex QTRAP 6500 LC-MS/MS platform. Three biological replicates were performed.

\section{RESULTS}

\section{Zma-unmiR4 is a novel maize miRNA in response to $F$. verticillioides}

Deep sequencing of small RNA libraries from maize kernels treated with or without $F$. verticillioides revealed a number of $F$. verticillioides -responsive miRNAs in our previous studies (Zhou et al., 2020), including 92 potentially novel miRNAs. These predicted miRNAs displayed various expression profiles in response to F. verticillioides (Figure 1A). A novel miRNA candidate designated zma-unmiR4 was characterized in more detail for its contrary expression in F. verticillioides susceptible maize line N6 and resistant line BT-1 (Figure 1B). Initially, successful amplification of its precursor sequence indicated that zma-unmiR4 is transcribed as an individual transcriptional unit in maize genome (Figure 1C). In addition, zma-unmiR4 was validated through RNA blotting in the maize kernels (Figure 1D). A high degree of complementarity for the precursor structure was further observed using RNAfold web server (Figure 1E). These observations support the 
notion that zma-unmiR4 represents a novel recently evolved miRNA potentially regulating the resistance to $F$. verticillioides in maize. Moreover, zma-unmiR4 was found to be expressed in various maize tissues (Figure S1), implying its potential functions during various developmental stages.

\section{ZmGA20x4 and its homolog AtGA2ox7 are the targets of zma-unmiR4}

Based on target gene prediction (http://rna.informatik.uni-freiburg.de), zma-unmiR4 showed extensive sequence complementarity with the geneZm00001d017294 encoding for gibberellin 2-oxidase 4 (ZmGA2ox4 ; Figure 2A). Notably, the accumulation of $Z m G A 20 x 4$ transcripts was drastically increased in BT-1 but decreased in N6 after $F$. verticillioides inoculation (Figure 2B), contrary to zma-unmiR4 expressions (Figure 1A-C). To confirm that $Z m G A 20 x 4$ is regulated by zma-unmiR4 in planta, the constructs respectively expressing zma-unmiR4 (35S:pre-unmiR4) and ZmGA20x4-YFP (35S: ZmGA2ox4-YFP) were co-transformed into maize protoplasts (Figure 2C), and YFP signals were significantly decreased in the protoplasts (Figure 2D). We further expressed 35S: ZmGA2ox4-GUS and 35S:pre-unmiR4 transgenes in tobacco leaves, and found that the GUS signals were nearly undetectable compared with the strong GUS staining when expressing 35S:ZmGA2ox4-GUSalone (Figure 2E). RT-qPCR also showed that ZmGA2ox4 transcripts were significantly decreased when co-expressing both transgenes (Figure 2F). Together, these results demonstrate that $Z m G A 20 x 4$ is a direct target of zma-unmiR4.

Arabidopsis AtGA2ox7 and AtGA2ox8 encoding for the homologous proteins of ZmGA2ox4 were predicted to be the putative heterologous targets of zma-unmiR4 (Figure 3A). We then compared the expression changes of AtGA2ox7 or AtGA2ox8 between wild type and zma-unmiR4 overexpressing (zma-unmiR4 OE ; Figure 3B) plants. As shown in Figure 3C, AtGA20x7 was remarkedly downregulated while AtGA2ox8 displayed no obvious changes in both zma-unmiR4 overexpressors, suggesting that AtGA2ox7 may be targeted by zma-unmiR4. To verify this regulation in planta, AtGA20x7 was fused with GUS gene and transiently co-expressed with 35S:pre-unmiR4 in tobacco. GUSactivities and transcripts were dramatically decreased compared with the vector control (Figure 3D and 3E), manisfested by the greatly reduction of AtGA2ox 7 transcripts (Figure 3F). These data demonstrate that zma-unmiR4 negatively regulates AtGA2ox7 in Arabidopsis .

\section{Overexpression of zma-unmiR4 confers Arabidopsis growth and F. verticillioides susceptibility}

To investigate the biological functions of zma-unmiR4, we developed homozygous transgenic Arabidopsis lines overexpressing zma-unmiR4 (zma-unmiR4 OE , Figure 3A),ZmGA2ox4 (ZmGA2ox4 OE ; Figure S2A), and AtGA2ox7 (AtGA2ox7 OE ; Figure S2B), respectively. Interestingly, we found that zma-unmiR 4 OEplants displayed increased plant height, early flowering, and large leaf size compared with WT plants, which were similar with the phenotypes ofatga2ox7 mutants (SALK_055721C; Figure 4A; Figure S3). On the contrary, ectopic expression of ZmGA20x4 or AtGA20x7 greatly reduced plant height, delayed flowering time, and shorten leaf radius, and the leaves of AtGA2ox7 OE appeared dark green with higher chlorophyll content (Figure 4A and 4B; Figure S3), as observed previously (Porri et al., 2012; Shu et al., 2016). In addition, exogenous application of bioactive GA could partially rescue the dwarf phenotype of ZmGA2ox4 OE and AtGA20x7 OEplants (Figure S4), implying the conserved functions of ZmGA20x4and AtGA2ox7 in GAmediated plant growth.

We then determined the $F$. verticillioides resistance of various genotypic plants by inoculating fungal spore suspension. On the 5th day post inoculation, young leaves of zma-unmiR4 OE andatga2ox7 mutant plants displayed obvious disease symptoms, and the size of yellow necrotic lesions were much larger than that of WT (Figure 4B). By contrast, the leaves of AtGA20x7 OE andZmGA2ox4 OE plants exhibited slight yellowish necrosis (Figure 4B). In addition, the rosette leaves of zma-unmiR $4 \mathrm{OE}$ and atga2ox 7 adult plants displayed severer blight or death phenotypes after spraying with $F$. verticillioides spore suspension, however, the transgenic plants of $Z m G A 20 x 4 O E$ or $A t G A 20 x^{\prime 7} O E$ were almost unaffected (Figure 4C).

We further tested whether there existed differences in F. verticillioides seed rot among WT, atga2ox7 mutant, and the transgenic plants indicated above. To this end, the seeds from various genotypes were incubated with $F$. verticillioides spore suspension, and the phenotypes of fungal mycelia growth on seeds surface were 
recorded on 6th day. Compared to the water-treatment control, the growth and invasion areas of fungal mycelia showed significant difference among various genotypes after $F$. verticillioidesinoculation. The seeds from zma-unmiR $4 \mathrm{OE}$ or atga2ox 7 mutant plants were more sensitive to $F$. verticillioides but resistant from ZmGA2ox4 OE or AtGA2ox7 OEtransgenic plants (Figure 4D). In details, more than half of the seeds from zma-unmiR4 $O E$ and atga2ox7 mutant plants exhibited disease grades II and III, however, most of seeds from $Z m G A 20 x 4 O E$ and AtGA20x7 $O E$ plants belonged to grade I according to the three grades of disease resistance (Figure S5). These data suggested that zma-unmiR4 could regulated plant growth positively and $F$. verticillioides resistance negatively by manipulating AtGA2ox7 or ZmGA2ox4 expression.

\section{Altered resistance to $F$. verticillioides by zma-unmiR4 is associated with the production of $\mathrm{H}_{2} \mathrm{O}_{2}$}

As one kind of necrotrophic fungal pathogens, F. verticillioides might ultimately kill and benefit from the infected host cells (Rivas-San Vicente et al., 2013). Leaves of various genotypic plants were incubated with 3,3'-diaminobenzidine (DAB) to detect $\mathrm{H}_{2} \mathrm{O}_{2}$ or stained with trypan blue (TB) to reveal dead cells. We first compared the $\mathrm{H}_{2} \mathrm{O}_{2}$ level and cell death between the leaves of $F$. verticillioides susceptible maize line $\mathrm{N} 6$ and resistant line BT-1 after F. verticillioides infection. Higher levels of $\mathrm{H}_{2} \mathrm{O}_{2}$ production and clusters of dead cells were observed in N6 leaves but less in BT-1 leaves (Figure 5A and 5B), implying that $F$. verticillioides hypersensitivity led to $\mathrm{H}_{2} \mathrm{O}_{2}$ burst and cell death in maize. Consistent with the differences of F. verticillioides resistance, we also noted higher $\mathrm{H}_{2} \mathrm{O}_{2}$ levels (Figure 5C) and more cell death (Figure 5D) in zma-unmiR4 $O E$ leaves compared with that of WT. On the contrary, the $\mathrm{H}_{2} \mathrm{O}_{2}$ accumulation and cell death were nearly undetectable in $Z m G A 20 x 4 O E$ and $A t G A 20 x 7 O E$ leaves (Figure $5 \mathrm{C}$ and 5D).

Development of $\boldsymbol{F}$. verticillioides resistance by zma-unmiR4 correlates with the expression of defense-related genes

We measured the relative levels of a set of defense-related genesPR1 , PR4 , PR5 ,PDF1.2 , NPR1, WRKY70, ORA59, and HCHIB/PR3 mRNAs in the transgenic plants indicated above. Notably, the expression levels of these defense-related genes were significantly induced in response to $F$. verticillioides infection in WT and AtGA2ox7 OEplants (Figure 6). In addition, all these genes except for PR5 were greatly upregulated in AtGA20x7 OE plants compared to the WT upon F. verticillioides infection (Figure 6). However, for zma-unmiR4 OE plants, the expression levels of PR1 ,PR4, PR5, and ORA59 displayed no obvious changes after $F$. verticillioides inoculation, and PDF1 ,NPR1, WRKY 70 , and $H C H I B$ were even downregulated after $F$. verticillioides inoculation (Figure 6). These data suggested that zma-unmiR4mediated suppression of AtGA20x7 might disturb the induction of defense genes by F. verticillioides, thus resulting in resistance variations.

\section{GA accumulation is associated with $F$. verticillioides resistance}

AtGA2ox7, a member of gibberellin 2-oxidase family, is a class of 2-oxoglutarate-dependent dioxygenases that regulate the deactivation of bioactive GAs (Li et al., 2019). We then analyzed the endogenous contents of bioactive GAs including GA1, GA3, GA4, and GA7 in the rosette leaves of four-week-old plants. The levels of GA3 and GA4 were too low to detect in WT, zma-unmiR4 OE , and AtGA2ox7OE samples tested, but GA1 were accumulated to higher levels inzma-unmiR4 OE plants than both WT and AtGA2oxy OEplants (Figure 7A). In addition, compared with WT, zma-unmiR4 OEtransgenic plants accumulated higher levels of GA7 while the contents of GA7 were significantly decreased in AtGA2ox7 OE (Figure 7B). These results suggested that zma-unmiR4-AtGA2ox7 module mediated plant growth and F. verticillioides resistance were more likely through regulating endogenous bioactive GAs accumulation.

To further investigate the effects of GA on plant disease resistance and growth, 17-day-old seedlings of WT, zma-unmiR4 $O E$ and AtGA2ox7 $O E$ transgenes were sprayed with GA $(50 \mu \mathrm{M})$ or GA synthesis inhibitor uniconazole $(20 \mu \mathrm{M})$. As expected, the growth of WT and AtGA2oxy $O E$ seedlings was enhanced by GA treatment, but growth inhibition was clearly observed for both the zma-unmiR4 $O E$ and WT plants when treated with uniconazole (Figure 8A). We then inoculated the leaves with F. verticillioides spore suspension. Compared to the water control treatment, the leaves of WT and AtGA2ox7 OE plants treated with GA 
displayed larger yellow necrotic lesions, and more $\mathrm{H}_{2} \mathrm{O}_{2}$ production as well as cell death (Figure 8B). By contrast, both WT and zma-unmiR4 OEtreated with uniconazole exhibited slight necrotic lesions, and the extents of $\mathrm{H}_{2} \mathrm{O}_{2}$ production and cell death were much lower than the non-treated control (Figure 8B).

Moreover, we applied GA or uniconazole on the susceptible maize line N6 to test the resistance changes to $F$. verticillioides . Compared with the application of water, the F. verticillioidessusceptibility of N6 seedlings was greatly promoted by GA, consistent with increased $F$. verticillioides -caused cell death and $\mathrm{H}_{2} \mathrm{O}_{2}$ accumulation (Figure 8C-E). By contrast, maize seedling treated with uniconazole displayed smaller necrotic lesions (Figure 8D), and decreased cell death and $\mathrm{H}_{2} \mathrm{O}_{2}$ production (Figure 8E). Furthermore, the similar results were also observed in rice seedlings treated with GA and GA inhibitors (Figure S6). When the rice seedlings were sprayed directly with $F$. verticillioides spore suspension, the disease symptom of the seedlings treated with GA was obvious enhanced, while seedlings treated with uniconazole was the opposite. Collectively, these results demonstrate that GA plays a negative role in plants resistance to $F$. verticillioides.

\section{DISCUSSION}

Fusarium verticillioides ( $F$. verticillioides ) is one of the most common pathogenic fungus that can cause many prevalent diseases in crops, especially for maize, such as seedling blight, root rot, stalk rot, ear rot, and seed rot, leading to poor grain yields and quality, thus posing a great challenge to food and feed safety (Gai et al., 2018; Ju et al., 2017; Mu et al., 2018; Septiani et al., 2019; Zhou et al., 2018). Identification of genes related to $F$. verticillioidesresistance and subsequent development of $F$. verticillioides -resistance crops are considered to be the most economical and environment-friendly strategy.

Given that miRNAs provide quantitative regulation of target gene expression rather than switching regulation, the dynamic accumulation of pathogen-responsive miRNAs can provide fine-tuning of target gene expression during pathogen infection, thus in turn enhancing the plant's disease-resist ability (Campo et al., 2013). High-throughput sequencing of small RNA is an effective method to discover pathogen-responsive miRNAs, including conserved and novel miRNAs. Although false-positive prediction of novel miRNAs cannot be ruled out during sequencing and data processing, the function of the young evolved miRNAs in pathogen resistance should be fully considered. For instance, the Md-miRln20 (Zhang et al., 2019), osa-miR7695 (Campo et al., 2013), and Md-miRLn11 (Ma et al., 2014) were characterized by small RNA sequencing and experimentally validated for their function in disease resistance. In the previous study, multiple $F$. verticillioides -responsive miRNAs were identified using small RNA deep sequencing (Zhou et al., 2020), and one of novel miRNAs zma-unmiR4 displayed entirely different expression patterns between the susceptible and resistant maize line after $F$. verticillioides infection (Figures 1A-C), and RNA blotting provided evidence for the existence of zma-unmiR4 in maize (Figure 1D). The significantly reduction of zma-unmiR4 in BT-1 upon F. verticillioides exposure indicated that it may function as a negative regulator of maize immunity against F. verticillioides (Figures 1A-C), manifested by the compromised resistance of transgenic plants ectopically expressing zma-unmiR4 in Arabidopsis (Figure 4).

According to the different life styles, plant pathogens can be divided into biotrophs (prefer living cells) and necrotrophs (prefer dead cells) (Barna et al., 2012). In the case of the necrotrophic pathogen such as Botrytis cinerea, ROS-overaccumulation-caused cell death and tissue necrosis during pathogen infection were reported to benefit the pathogen invasion by offering a growth substrate, thus increasing host susceptibility (Hanif et al., 2018; Tian et al., 2019; Wang et al., 2018). F. verticillioides was reported to be one kind of necrotrophic fungal pathogens (Rivas-San Vicente et al., 2013). In line with this, more cell death and $\mathrm{H}_{2} \mathrm{O}_{2}$ production were detected in the leaves of susceptible maize line N6 compared with the resistant maize line BT-1 after $F$. verticillioides infection (Figure 5). Similarly, the susceptibility to $F$. verticillioides infection was also correlated with the levels of cell death and $\mathrm{H}_{2} \mathrm{O}_{2}$ production in WT,zma-unmiR4 OE, AtGA2ox7 OE and $Z m G A 20 x 4$ OE plant (Figure 4 and Figure 5). Therefore, cell death and $\mathrm{H}_{2} \mathrm{O}_{2}$ accumulation can be used as the indicators of $F$. verticillioides susceptibility in maize cultivars.

Gibberellins are widely recognized as phytohormones that play multiple roles in plant development and stress responses (Rizza and Jones, 2019; Schomburg et al., 2003). Endogenous levels of bioactive GAs are 
maintained through a balance of biosynthesis and inactivation. AtGA2ox7 is a class of 2-oxoglutaratedependent dioxygenases that regulate the deactivation of bioactive GAs ( $\mathrm{Li}$ et al., 2019). Consistently, the transgenic plants overexpressing AtGA2ox7 showed significant reduction of bioactive GAs compared to the WT, thus exhibiting GA-deficient phenotypes, such as dwarf plant, delayed flowering, and small dark green leaves (Porri et al., 2012; Schomburg et al., 2003; Shu et al., 2016) (Figure 4A and 4B; Figure 7; Figure S3). By contrast, dysfunction of AtGA2ox7 resulted in GA-induced phenotypes, including enhanced growth, large leaf size, and early flowering(Magome et al., 2008; Rieu et al., 2008; Shu et al., 2016), which was consistent with the phenotypes of zma-unmiR4 OEplants and higher levels of GA contents (Figure 4A and 4B; Figure 7; Figure S3). Therefore, we have reason to believe that the high level of bioactive GAs by zma-unmiR4-mediated repression of AtGA2ox7 is responsible for the phenotypic changes of zma-unmiR4 $O E$ plants.

Although the function of bioactive GA in plant growth and development is well reported, the role of GA in plant resistance to $F$. verticillioides remains unclear. In fact, GA was first identified from Gibberella fujikuroi (Fusarium moniliforme), one kind of necrotrophic fungus that causes rice bakanae disease (Yabuta and Sumiki, 1938). Overexpression of the GA deactivating enzyme Eui could increase the resistance to bacterial blight and rice blast respectively caused by Xanthomonas oryzae and Magnaporthe oryzae, however, transgenic rice overexpressing OsGA20ox3 (encoding for a GA biosynthetic enzyme) displayed hypersensitivity to both diseases (Yang et al., 2008; Qin et al., 2013). Similarly, our current results demonstrated that GAs also exhibited a negative effect on the resistance to $F$. verticillioides through genetic and physiological analysis in Arabidopsis or maize plants (Figures 4 and 8). Engineering the expression level of GA-deactivating enzyme AtGA20x7 or ZmGA20x4 could modify the F. verticillioides resistance (Figure 4). Despite of the enhanced resistance to $F$. verticillioides in Arabidopsis with overaccumulation of AtGA2ox 7 , many adverse effects were also occurred on development, such as dwarf plants and delayed flowering (Figure 4A), which would be likely expected in maize. In addition, it has been reported that application of GA inhibitor uniconazole significantly reduced lodging rate and enhanced yield in maize (Ahmad et al., 2021). Our data also showed the positive effects of uniconazole on $F$. verticillioidesresistance and dwarf traits in maize and rice (Figure $8 \mathrm{C}-\mathrm{E}$ and Figure S6). Therefore, fine-tuning zma-unmiR4-ZmGA2ox4 regulatory module could theoretically be an alternative way to generate desirable $F$. verticillioides and lodging resistance without growth or yield penalty in maize breeding.

Given the crucial role of GA in plant innate immunity (De Vleesschauwer et al., 2016; Qin et al., 2013; Yang et al., 2008), it is not surprising to find the divergent resistance to $F$. verticillioides between the GAdeficient (AtGA2ox7 OE) and GA-sufficient (zma-unmiR4 OE) plants (Figure 4, 5, 8). On the one hand, GA may give rise to an indirect attenuation of pathogenesis-related (PR) genes, thus facilitating pathogen prevalence. Indeed, the expression levels of $P R 1, P R 3, P R 4$, and PR5 were significantly induced in response to $F$. verticillioides infection in AtGA2ox7 OE plants, however, they displayed no obvious changes after $F$. verticillioides inoculation in zma-unmiR4 $O E$ (Figure 6). On the other hand, GA may hinder disease defense responses via modulating the homeostasis of the archetypal immunity hormones (Verma et al., 2016; Wild and Achard, 2013), such as salicylic acid (SA), jasmonic acid (JA), and ethylene (ET). In rice, overexpression or dysfunction of GA deactivating enzyme Eui results in disturbed homeostasis of SA and JA, thus leading to the altered disease susceptibility (Yang et al., 2008). In addition, the SA receptors non-expresser of pathogenesis-related genes $1(N P R 1)$ is a master regulator of systemic acquired resistance in plants, overaccumulation of NPR1 leads to enhanced disease resistance to diverse pathogens (Ding et al., 2018). In support of this notion, we found that NPR1 was significantly induced after $F$. verticillioides infection in AtGA2ox7OE plants but repressed in zma-unmiR4 OE plants (Figure 6), implying divergent SA signaling dynamics in those transgenic plants upon pathogen attack. Moreover, NPR1 might activate several WRKY transcription factors, such as WRKY70, subsequently leading to massive induction of antimicrobial genes (Saleh et al., 2015). As expected, F. verticillioides -induced expressions of WRKY70in the WT, zma-unmiR 4 OE and AtGA2ox7 OE plants was exactly similar with that of NPR1 (Figure 6). Additionally, the divergent expression profiles of ORA59 and the JA- and ET-responsive plant defensin gene PDF1.2(Zarei et al., 2011) further revealed the different dynamics of JA- and ET-associated resistant 
responses in the transgenic plants upon $F$. verticillioidesexposure. Further genome-wide transcriptome analysis with thezma-unmiR4 $O E$ and AtGA2ox7 OE plants should provide much-needed insights into to the interactions between GA and other phytohormones signaling pathways that underpin plant resistance in response to $F$. verticillioides challenging.

Under stress conditions, plants are believed to actively repress their growth for survival, but accompanied with undesirable crop productivity (Zhang et al., 2020). As a master regulator of plant growth and development, however, GA confers susceptibility to multiple plant diseases (Qin et al., 2013; Yang et al., 2008). Overall, our results revealed the novel miRNA zma-unmiR4 played as a crucial regulator inF. verticillioides resistance and plant growth by suppressing bioactive GA accumulation (Figure 9). Thus, engineering the zma-unmiR4-ZmGA20x4 module may thereby represent an alternative strategy that warrants a better balance between $F$. verticillioides disease resistance and growth in corn breeding.

\section{ACKNOWLEDGEMENTS}

This work was supported by the Joint Funds of the National Natural Science Foundation of China (Grant No. U2004157 to H.Z), the International (Regional) Joint Research Project of the National Natural Science Foundation of China (Grant No. 31761143009 to J.W), and the Innovation Special Program of Henan Agricultural University for Science and Technology (Grant No. 30501044 to Y.X.).

\section{CONFLICT OF INTEREST}

The authors declare no conflicts of interest.

\section{REFERENCES}

Ahmad, I., Ahmad, S., Yang, X.N., Meng, X.P., Yang, B.P., Liu, T., and Han, Q.F. (2021). Effect of uniconazole and nitrogen level on lodging resistance and yield potential of maize under medium and high plant density. Plant Biol. 23: 485-496.

Arnon, D.I. (1949). Copper enzymes in isolated chloroplasts polyphenoloxidase in beta vulgaris. Plant Physiol. 24: 1-15.

Barna, B., Fodor, J., Harrach, B.D., Pogány, M., and Király, Z. (2012). The Janus face of reactive oxygen species in resistance and susceptibility of plants to necrotrophic and biotrophic pathogens. Plant Physiol. Biochem. 59: 37-43.

Butrón, A., Santiago, R., Cao, A., Samayoa, L.F., and Malvar, R.A. (2019). QTLs for resistance to Fusarium ear rot in a multiparent advanced generation intercross (MAGIC) maize population. Plant Dis. 103: 897-904.

Campo, S., Peris-Peris, C., Siré, C., Moreno, A.B., Donaire, L., Zytnicki, M., Notredame, C., Llave, C., and San Segundo, B. (2013). Identification of a novel microRNA (miRNA) from rice that targets an alternatively spliced transcript of the Nramp6 (Natural resistance-associated macrophage protein 6) gene involved in pathogen resistance. New Phytol. 199: 212-227.

Chen, J., Shrestha, R., Ding, J., Zheng, H., Mu, C., Wu, J., and Mahuku, G. (2016). Genome-wide association study and QTL mapping reveal genomic loci associated with Fusarium ear rot resistance in tropical maize germplasm. G3 6: 3803-3815.

Chen, J.F., Zhao, Z.X., Li, Y., Li, T.T., Zhu, Y., Yang, X.M., Zhou, S.X., Wang, H., Zhao, J.Q., Pu, M., et al. (2021). Fine-tuning roles of Osa-miR159a in rice immunity against Magnaporthe oryzae and development. Rice 14: 26.

De Vleesschauwer, D., Seifi, H.S., Filipe, O., Haeck, A., Huu, S.N., Demeestere, K., and Höfte, M. (2016). The DELLA protein SLR1 integrates and amplifies salicylic acid-and jasmonic acid-dependent innate immunity in rice. Plant Physiol. 170: 1831-1847.

Ding, Y., Sun, T., Ao, K., Peng, Y., Zhang, Y., Li, X., and Zhang, Y. (2018). Opposite roles of salicylic acid receptors NPR1 and NPR3/NPR4 in transcriptional regulation of plant immunity. Cell 173: 1454-1467. 
Feng, Q., Li, Y., Zhao, Z.X., and Wang, W.M. (2021). Contribution of small RNA pathway to interactions of rice with pathogens and insect pests. Rice 14: 15.

Gai, X., Dong, H., Wang, S., Liu, B., Zhang, Z., Li, X., and Gao, Z. (2018). Infection cycle of maize stalk rot and ear rot caused by Fusarium verticillioides . PLoS One 13: e0201588.

Hanif, M., Rahman, M.U., Gao, M., Yang, J., Ahmad, B., Yan, X., and Wang, X. (2018). Heterologous expression of the grapevine $J A Z 7$ gene in Arabidopsis confers enhanced resistance to powdery mildew but not to Botrytis cinerea . Int. J. Mol. Sci. 19: 3889.

Hu, G., Lei, Y., Liu, J., Hao, M., Zhang, Z., Tang, Y., Chen, A., and Wu, J. (2020). The ghr-miR164 and GhNAC100 modulate cotton plant resistance against Verticillium dahlia . Plant Sci. 293: 110438.

Ju, M., Zhou, Z., Mu, C., Zhang, X., Gao, J., Liang, Y., Chen, J., Wu, Y., Li, X., Wang, S., et al. (2017). Dissecting the genetic architecture of Fusarium verticillioides seed rot resistance in maize by combining QTL mapping and genome-wide association analysis. Sci. Rep. 7: 46446.

Kumar, R. (2014). Role of microRNAs in biotic and abiotic stress responses in crop plants. Appl. Biochem. Biotechnol. 174: 93-115.

Lanubile, A., Maschietto, V., Borrelli, V.M., Stagnati, L., Logrieco, A.F., and Marocco, A. (2017). Molecular basis of resistance toFusarium ear rot in maize. Front. Plant Sci. 8: 1774.

Li, C., Wong, A.Y.P., Wang, S., Jia, Q., Chuang, W.P., Bendena, W.G., Tobe, S.S., Yang, S.H., Chung, G., Chan, T.F., et al. (2018). miRNA-mediated interactions in and between plants and insects. Int. J. Mol. Sci. 19: 3239 .

Li, C., Zheng, L., Wang, X., Hu, Z., Zheng, Y., Chen, Q., Hao, X., Xiao, X., Wang, X., Wang, G., et al. (2019). Comprehensive expression analysis of Arabidopsis GA2-oxidase genes and their functional insights. Plant Sci. 285: 1-13.

Li, T., Lian, H., Li, H., Xu, Y., and Zhang, H. (2021). HY5 regulates light-responsive transcription of microRNA163 to promote primary root elongation in Arabidopsis seedlings. J. Integr. Plant Biol. 63: 14371450 .

Li, Y., Lu, Y.G., Shi, Y., Wu, L., Xu, Y.J., Huang, F., Guo, X.Y., Zhang, Y., Fan, J., Zhao, J.Q., et al. (2014). Multiple rice microRNAs are involved in immunity against the blast fungus Magnaporthe oryzae . Plant Physiol. 164: 1077-1092.

Liu, J.X., Cai, Y.N., Jiang, W.Y., Li, Y.G., Zhang, Q.F., and Pan, H.Y. (2020). Population structure and genetic diversity of fungi causing rice seedling blight in northeast China based on microsatellite markers. Plant Dis. 104: 868-874.

Liu, M., Shi, Z., Zhang, X., Wang, M., Zhang, L., Zheng, K., Liu, J., Hu, X., Di, C., Qian, Q., et al. (2019). Inducible overexpression of Ideal Plant Architecture1 improves both yield and disease resistance in rice. Nat. Plants 5: 389-400.

Ma, C., Lu, Y., Bai, S., Zhang, W., Duan, X., Meng, D., Wang, Z., Wang, A., Zhou, Z., and Li, T. (2014). Cloning and characterization of miRNAs and their targets, including a novel miRNA-targeted NBS-LRR protein class gene in apple (Golden Delicious). Mol. Plant. 7: 218-230.

Machado, J., Machado, A.Q., Pozza, E.A., Machado, C.F., and Zancan, W. (2013). Inoculum potential of Fusarium verticillioides and performance of maize seeds. Trop. Plant Pathol. 38: 213-217.

Magome, H., Yamaguchi, S., Hanada, A., Kamiya, Y., and Oda, K. (2008). The DDF1 transcriptional activator upregulates expression of a gibberellin-deactivating gene, GA20x7, under high-salinity stress in Arabidopsis . Plant J. 56: 613-626. 
Maschietto, V., Colombi, C., Pirona, R., Pea, G., Strozzi, F., Marocco, A., Rossini, L., and Lanubile, A. (2017). QTL mapping and candidate genes for resistance to Fusarium ear rot and fumonisin contamination in maize. BMC Plant Biol. 17: 20.

Mengistu, A.A., and Tenkegna, T.A. (2021). The role of miRNA in plant-virus interaction: a review. Mol. Biol. Rep. 48: 2853-2861.

Mu, C., Gao, J., Zhou, Z., Wang, Z., Sun, X., Zhang, X., Dong, H., Han, Y., Li, X., Wu, Y., et al. (2018). Genetic analysis of cob resistance to $F$. verticillioides : another step towards the protection of maize from ear rot. Theor. Appl. Genet. 132: 1049-1059.

Navarro, L., Dunoyer, P., Jay, F., Arnold, B., Dharmasiri, N., Estelle, M., Voinnet, O., and Jones, J.D. (2006). A plant miRNA contributes to antibacterial resistance by repressing auxin signaling. Science 312: 436-439.

Porri, A., Torti, S., Romera-Branchat, M., and Coupland, G. (2012). Spatially distinct regulatory roles for gibberellins in the promotion of flowering of Arabidopsis under long photoperiods. Development 139: 2198-2209.

Qin, X., Liu, J.H., Zhao, W.S., Chen, X.J., Guo, Z.J., and Peng, Y.L. (2013). Gibberellin 20-oxidase gene OsGA20ox3 regulates plant stature and disease development in rice. Mol. Plant Microbe Interact. 26: 227239.

Rieu, I., Eriksson, S., Powers, S.J., Gong, F., Griffiths, J., Woolley, L., Benlloch, R., Nilsson, O., Thomas, S.G., Hedden, P., et al. (2008). Genetic analysis reveals that C19-GA 2-oxidation is a major gibberellin inactivation pathway in Arabidopsis . Plant Cell 20: 2420-2436.

Rivas-San Vicente, M., Larios-Zarate, G., and Plasencia, J. (2013). Disruption of sphingolipid biosynthesis in Nicotiana benthamianaactivates salicylic acid-dependent responses and compromises resistance to Alternaria alternata f. sp. lycopersici. Planta 237: 121-136.

Rizza, A., and Jones, A.M. (2019). The makings of a gradient: spatiotemporal distribution of gibberellins in plant development. Curr. Opin. Plant Biol. 47: 9-15.

Rosa Junior, O.F., Dalcin, M.S., Nascimento, V.L., Haesbaert, F.M., Ferreira, T.P.S., Fidelis, R.R., Sarmento, R.A., Aguiar, R.W.S., Oliveira, E.E., and Santos, G.R.D. (2019). Fumonisin production by Fusarium verticillioides in maize genotypes cultivated in different environments. Toxins 11: 215.

Rubio-Somoza, I., and Weigel, D. (2011). MicroRNA networks and developmental plasticity in plants. Trends Plant Sci. 16: 258-264.

Saleh, A., Withers, J., Mohan, R., Marqués, J., Gu, Y., Yan, S., Zavaliev, R., Nomoto, M., Tada, Y., and Dong, X. (2015). Posttranslational modifications of the master transcriptional regulator NPR1 enable dynamic but tight control of plant immune responses. Cell host \& microbe 18: 169-182.

Schiwek, S., Beule, L., Vinas, M., Pfordt, A., von Tiedemann, A., and Karlovsky, P. (2020). High-resolution melting (HRM) curve assay for the identification of eight Fusarium species causing ear rot in maize. Pathogens 9: 270 .

Schomburg, F.M., Bizzell, C.M., Lee, D.J., Zeevaart, J.A., and Amasino, R.M. (2003). Overexpression of a novel class of gibberellin 2-oxidases decreases gibberellin levels and creates dwarf plants. Plant Cell 15: 151-163.

Septiani, P., Lanubile, A., Stagnati, L., Busconi, M., Nelissen, H., Pe, M.E., Dell'Acqua, M., and Marocco, A. (2019). Unravelling the genetic basis of Fusarium seedling rot resistance in the MAGIC maize population: novel targets for breeding. Sci. Rep. 9: 5665.

Shu, K., Chen, Q., Wu, Y., Liu, R., Zhang, H., Wang, P., Li, Y., Wang, S., Tang, S., Liu, C., et al. (2016). ABI4 mediates antagonistic effects of abscisic acid and gibberellins at transcript and protein levels. Plant J. 
85: $348-361$.

Stagnati, L., Lanubile, A., Samayoa, L.F., Bragalanti, M., Giorni, P., Busconi, M., Holland, J.B., and Marocco, A. (2019). A genome wide association study reveals markers and genes associated with resistance to Fusarium verticillioides infection of seedlings in a maize diversity panel. G3 9: 571-579.

Sunkar, R., Li, Y.F., and Jagadeeswaran, G. (2012). Functions of microRNAs in plant stress responses. Trends Plant Sci. 17: 196-203.

Tian, S., Yin, X., Fu, P., Wu, W., and Lu, J. (2019). Ectopic expression of grapevine gene VaRGA1 in Arabidopsis improves resistance to downy mildew and Pseudomonas syringae pv. tomato DC3000 but increases susceptibility to Botrytis cinerea. Int. J. Mol. Sci. 21: 193.

Verma, V., Ravindran, P., and Kumar, P.P. (2016). Plant hormone-mediated regulation of stress responses. BMC Plant Biol. 16: 86.

Voinnet, O. (2009). Origin, biogenesis, and activity of plant microRNAs. Cell 136: 669-687.

Wang, M., Zhu, Y., Han, R., Yin, W., Guo, C., Li, Z., and Wang, X. (2018). Expression of vitis amurensis VaERF20 inArabidopsis thaliana improves resistance to Botrytis cinerea and Pseudomonas syringae pv. Tomato DC3000. I. J. Mol. Sci. 19: 696.

Wang, Y., Zhou, Z., Gao, J., Wu, Y., Xia, Z., Zhang, H., and Wu, J. (2016). The mechanisms of maize resistance to Fusarium verticillioides by comprehensive analysis of RNA-seq data. Front. Plant Sci. $7: 1654$.

Wild, M., and Achard, P. (2013). The DELLA protein RGL3 positively contributes to jasmonate/ethylene defense responses. Plant Signal. Behav. 8: e23891.

Wu, J., Yang, R., Yang, Z., Yao, S., Zhao, S., Wang, Y., Li, P., Song, X., Jin, L., Zhou, T., et al. (2017). ROS accumulation and antiviral defence control by microRNA528 in rice. Nat. Plants 3: 16203.

Wu, Y., Zhou, Z., Dong, C., Chen, J., Ding, J., Zhang, X., Mu, C., Chen, Y., Li, X., Li, H., et al. (2020). Linkage mapping and genome-wide association study reveals conservative QTL and candidate genes for Fusarium rot resistance in maize. BMC Genomics 21: 357.

Yabuta, T., and Sumiki, Y. (1938). On the crystal of gibberellin, a substance to promote plant growth. J. Agric. Chem. Soc. Japan 14: 1526.

Yang, D.L., Li, Q., Deng, Y.W., Lou, Y.G., Wang, M.Y., Zhou, G.X., Zhang, Y.Y., and He, Z.H. (2008). Altered disease development in the eui mutants and Eui overexpressors indicates that gibberellins negatively regulate rice basal disease resistance. Mol. Plant. 1: 528-537.

Yang, J., Zhang, F., Li, J., Chen, J.P., and Zhang, H.M. (2016). Integrative analysis of the microRNAome and transcriptome illuminates the response of susceptible rice plants to rice stripe virus. PLoS One 11: e0146946.

Yao, L., Li, Y., Ma, C., Tong, L., Du, F., and Xu, M. (2020). Combined genome-wide association study and transcriptome analysis reveal candidate genes for resistance to Fusarium ear rot in maize. J. Integr. Plant Biol. 62: 1535-1551.

Yao, S., Yang, Z., Yang, R., Huang, Y., Guo, G., Kong, X., Lan, Y., Zhou, T., Wang, H., Wang, W., et al. (2019). Transcriptional regulation of miR528 by OsSPL9 orchestrates antiviral response in rice. Mol. Plant 12: $1114-1122$.

Zarei A, Körbes AP, Younessi P, Montiel G, Champion A, Memelink J. (2011). Two GCC boxes and AP2/ERF-domain transcription factor ORA59 in jasmonate/ethylene-mediated activation of the PDF1.2 promoter in Arabidopsis . Plant Mol. Biol. 75: 321-31.

Zhang, H., and Li, L. (2013). SQUAMOSA promoter binding protein-like7 regulated microRNA408 is required for vegetative development inArabidopsis . Plant J. 74: 98-109. 
Zhang, H., Zhao, Y., and Zhu, J.K. (2020). Thriving under stress: how plants balance growth and the stress response. Dev. Cell 55: 529-543.

Zhang, T., Zhao, Y.L., Zhao, J.H., Wang, S., Jin, Y., Chen, Z.Q., Fang, Y.Y., Hua, C.L., Ding, S.W., and Guo, H.S. (2016). Cotton plants export microRNAs to inhibit virulence gene expression in a fungal pathogen. Nat. Plants 2: 16153.

Zhang, X., Zhao, H., Gao, S., Wang, W.C., Katiyar-Agarwal, S., Huang, H.D., Raikhel, N., and Jin, H. (2011). Arabidopsis Argonaute 2 regulates innate immunity via miRNA393(*)-mediated silencing of a Golgi-localized SNARE gene, MEMB12. Mol. Cell 42: 356-366.

Zhang, Y., Zhang, Q., Hao, L., Wang, S., Wang, S., Zhang, W., Xu, C., Yu, Y., and Li, T. (2019). A novel miRNA negatively regulates resistance to Glomerella leaf spot by suppressing expression of an $N B S$ gene in apple. Hortic. Res. 6: 93.

Zhou, B., Zhou, Z., Ding, J., Zhang, X., Mu, C., Wu, Y., Gao, J., Song, Y., Wang, S., Ma, J., et al. (2018). Combining three mapping strategies to reveal quantitative trait loci and candidate genes for maize ear length. Plant Genome 11: 170107.

Zhou, Z., Cao, Y., Li, T., Wang, X., Chen, J., He, H., Yao, W., Wu, J., and Zhang, H. (2020). MicroRNAs are involved in maize immunity against Fusarium verticillioides ear rot. Genom. Proteom. Bioinf. 18: $241-255$.

\section{Figure Legends}

\section{Figure 1. Identification and validation of zma-unmiR4}

(A ) Expression heatmap of the 92 predicted novel miRNAs candidates in BT-1 and N6 kernels after $F$. verticillioidesinoculation by small RNA sequencing (Zhou et al., 2020). Maize kernels of BT-1 and N6 at $0 \mathrm{~d}$ or $3 \mathrm{~d}$ post inoculation were sampled for small RNA libraries construction. (B ) Distinct expression of zma-unmiR4 in BT-1 and N6 maize lines after F. verticillioides inoculation by RT-qPCR. Maize Ef1a was used as the internal control, and the expression level of N6 on day 0 was set to 1 . Data are means +- SD from three biological replicates. ${ }^{* *} P<0.01$ by student'st -test. $(\mathbf{C}$ and $\mathbf{D})$ Verification of zma-unmiR4 through RT-PCR amplifying its precursor (C) and RNA blotting (D). (E ) Predicted hairpin structure of zma-unmiR4 by RNAfold software.

\section{Figure 2. Maize $Z m G A 2 o x 4$ is targeted by zma-unmiR4.}

(A ) Schematic diagram of ZmGA2ox4 showing the target site of zma-unmiR4. (B ) ZmGA2ox4 was differentially regulated in BT-1 and N6 kernels after F. verticillioidesinoculation. Maize Ef1a was used as the internal control, and the expression level of N6 on day 0 was set to 1. Data are means +- SD from three biological replicates. (C) Structure of various constructs used in the transient transformation assay in maize protoplast and tobacco plants. (D ) Maize protoplasts were transfected with the reporter plasmid with empty effector or35S:pre-unmiR4 effector, then the protoplasts were kept in darkness for $16 \mathrm{~h}$. The fluorescence intensity of YFP was normalized to the mCherry. Data are represented as mean +- SD (n [?] 200 cells) from three biological replicates. ( $\mathbf{E}$ and $\mathbf{F}$ ) Tobacco leaves were transfected with 35S: ZmGA2ox4-GUS reporter plasmid with empty effector or 35S:pre-unmiR4 effector. After two days, the transfected leaves were used for GUS staining (E) and isolation of total RNA for detecting ZmGA20x4 expression by RT-qPCR (F). Data are means $+-\mathrm{SD}$ from three biological replicates. ${ }^{* *} P<0.01$ by student's $t$-test.

Figure 3. Arabidopsis AtGA2ox7 is a heterologous target of zma-unmiR4.

(A ) Phylogenetic tree of ZmGA2ox4 homologs in maize andArabidopsis. The sequences of ZmGA2ox4 homologs from maize and Arabidopsis were obtained from NCBI database searches (https://blast.ncbi.nlm.nih.gov), and phylogenetic analysis was performed using MEGA7.0. The neighborjoining method was used with 1,000 bootstrap replications. (B ) Measurement of zma-unmiR4 precursor 
enrichment in two independent transgenic homozygous lines (zma-unmiR4 $O E$ ) by RT-PCR. (C) Expression levels of AtGA2ox 7 or AtGA2ox8 were quantified in WT and zma-unmiR4 OE plants by RT-qPCR. (D) GUS staining in tobacco leaves co-transformed with35S:AtGA2ox 7 -GUS construct and empty vector or the35S:pre-unmiR4 effector as mentioned in panel C of Figure 2. (E and $\mathbf{F}$ ) Quantification of GUS (E) and AtGA2ox7 (F) transcripts in the samples described in panel D. Data are means +- SD of three biological replicates. ${ }^{* *} P<0.01$ by Student's $t$-test; ns, no significant difference.

Figure 4. Zma-unmiR4 regulates $F$. verticillioidesresistance negatively and growth positively through the target geneAtGA2ox7 or ZmGA2ox4.

(A ) Growth phenotypes of WT, atga2ox7 mutant,zma-unmiR4 OE , AtGA2ox7 OE , and ZmGA2ox4 $O E$ plants. Four-week-old seedlings grown in soil were photographed. (B ) The disease symptoms on the representative leaves of WT, AtGA2ox 7mutant, zma-unmiR 4 OE, AtGA2ox7 OE, and ZmGA2ox4 OE plants at 5 days post inoculation. Healthy rosette leaves of 4 -week-old plants were inoculated with $20 \mu l F$. verticillioides spore suspension ( F. V) or sterile water (Mock). (C) The disease symptoms of WT, atga2ox 7 mutant, zma-unmiR4 $O E$, AtGA2ox7 $O E$, and $Z m G A 20 x 4 O E$ plants after F. verticillioides spraying. Fourweek-old plants grown in soil were sprayed with $F$. verticillioides spore suspension or sterile water. (D) The seed rot symptoms of WT, atga2oxy mutant, zma-unmiR4 OE ,AtGA2ox7 OE, and ZmGA2ox4 OE plants. Healthy dry seeds were sterilized, then immersed in $F$. verticillioides spore suspension for $48 \mathrm{~h}$ and placed in sterile filter paper for 6 days.

Figure 5. Fusarium verticillioides susceptibility is proceeded by $\mathrm{H}_{2} \mathrm{O}_{2}$ accumulation.

(A and $\mathbf{B}$ ) $\mathrm{H}_{2} \mathrm{O}_{2}$ production and cell death in maize leaves were revealed by diaminobenzidine (DAB) straining (A) and trypan blue (TB) straining (B), respectively. Healthy second leaves of BT-1 and N6 maize lines were injected with $F$. verticillioides spore suspension $(F . V)$ or sterile water (Mock), and the leaves were sampled for staining 2 days after inoculation. (C and D ) DAB staining (C) and TB staining (D) analysis in Arabidopsis leaves of indicated genotypic plants. Healthy rosette leaves of 4 -week-old plants were injected with $F$. verticillioides spore suspension or sterile water, and were sampled for straining 4 days after inoculation. The amplification and spread of $\mathrm{H}_{2} \mathrm{O}_{2}$ production and cell death in the whole leaf were shown in a large section of a leaf at $\times 5$ magnification.

Figure 6. Expression of the defense-related genes in transgenic Arabidopsis.

Healthy leaves of 4-week-old WT, zma-unmiR4 OE , andAtGA2ox7 OE plants were inoculated with $F$. verticillioidesspore suspension, and were sampled at indicated time point for total RNA extraction. Gene expression levels were quantified by RT-qPCR.Actin 2 was used as an internal control. Expression level in $\mathrm{WT}$ at $0 \mathrm{~h}$ was set to 1 . Data are means $\pm \mathrm{SD}$ of three biological replicates.

Figure 7. Quantification of endogenous bioactive GAs in WT, zma-unmiR4 OE, and AtGA2ox7 $O E$ plants.

Shoots of 4-week-old various genotypic plants were sampled for quantifying GA contents. Data are means \pm SD of three biological replicates. ${ }^{* *} P<0.01$ by Student's $t$-test.

Figure 8.Exogenous application of GA or GA inhibitors alters plant resistance to $F$. verticillioides

(A ) Growth phenotypes of WT, zma-unmiR4 $O E$ and AtGA2ox7 OE plants. 17-day-old indicated plants were sprayed with $\mathrm{H}_{2} \mathrm{O}$, GA $(50 \mu \mathrm{M})$ or uniconazole $(20 \mu \mathrm{M})$ once a day for 5 days, then photographed. (B ) The disease symptoms (top), DAB staining (middle), and TB staining (bottom) of the representative leaves from WT, zma-unmiR4 OE and AtGA2ox7OE plants. Healthy leaves of 17-day-old seedlings treated as above were inoculated with $F$. verticillioides spore suspension, then photographed or stained 4 days after inoculation. (C) Growth phenotypes of susceptible maize line $\mathrm{N} 6$ after exogenous spraying of $\mathrm{H}_{2} \mathrm{O}$, GA $(50 \mu \mathrm{M})$ or uniconazole $(20 \mu \mathrm{M})$. (D and $\mathbf{E})$ The disease symptoms (D), TB straining (E, left), and DAB straining (E, right) of $\mathrm{N} 6$ leaves. 7-days seedlings were sprayed with $\mathrm{H}_{2} \mathrm{O}, \mathrm{GA}(50 \mu \mathrm{M})$ or uniconazole $(20$ 
$\mu \mathrm{M})$ once a day for 7 days, then inoculated with $F$. verticillioides $(F . V)$ spore suspension or sterile water (Mock), and photographed or stained at 4 days after inoculation.

Figure 9. A hypothetical model of zma-unmiR4 in mediating plant resistance to $F$. verticillioides and growth.

zma-unmiR4 suppresses $Z m G A 20 x 4$ or AtGA20x7 expression by cleaving their transcripts, leading to bioactive gibberellin (GA) accumulation. On the one hand, increased GA perturbs JA/SA-mediated defense signaling, thereby resulting in compromised $F$. verticillioides resistance. On the other hand, increased bioactive GA confers plant growth and development. Thus, manipulating the zma-unmiR4- ZmGA2ox4 module may represent an alternative strategy that warrants a better balance between $F$. verticillioides disease resistance and growth in maize.

\section{Supplemental Information}

Figure S1. Tissue-specific expression of zma-unmiR4.

YR, YS and YL represented roots, stem and leaves from 8-day-old B73 seedlings, respectively, and stem and leaf were taken from the plants at the flowering stage. Total RNA was extracted from indicated tissues and treated with DNase I, and reverse-transcribed into cDNA for PCR amplification. Ef1a was used as a control.

Figure S2. Molecular identification of $Z m G A 20 x 4 O E$ and AtGA 2ox7 OE transgenic lines.

Leaves of 4-week-old $Z m G A 2 o x 4 O E$ and AtGA2ox7 OEtransgenic plants were sampled for total RNA extraction, and RT-qPCR assays were performed to measure the transcript levels of ZmGA2ox4 (A) and AtGA2ox 7 (B) genes. Actin2 was used as an internal control. Data are means \pm SD of three biological replicates. ${ }^{* *}$ $P<0.01$ by Student's $t$-test.

Figure S3. Quantitative comparison of flowering time, plant height, leaf size andchlorophyll contents among WT, atga2ox'7 mutant, and transgenic plants.

The timing of the first opened flower (A), rosette leaf number at time of the first open flower (B), plant height $(\mathrm{C})$, leaf size (D), leaf area (E), and chlorophyll concentration (F) of WT, atga2ox7 mutant, zma-unmiR4 OE , AtGA2ox7 $O E$, and ZmGA2ox4 OE plants. Four-week-old seedlings grown in soil were used for analysis. Data are means \pm SD. Panel A and $\mathrm{B}, \mathrm{n}=24$ plants; Panel $\mathrm{C}, \mathrm{n}=20$ plants; Panel E, the largest rosette leaf was used for measuring leaf area by Image $J$ software, $n=18$ plants. Panel $\mathrm{F}, \mathrm{n}=$ three biological replicates. ns, no significant difference; ${ }^{*} P<0.05,{ }^{* *} P<0.01$ by Student's $t$-test.

Figure S4. Exogenous GA partially rescued the dwarf phenotype by AtGA2ox7 or ZmGA2ox4 overaccumulation inArabidopsis.

Four-week-old AtGA2ox7 OE and ZmGA2ox4 OE transgenic plants grown in soil were sprayed with GA (50 $\mu \mathrm{M})$ once a day for 10 days, and then photographed.

Figure S5. Investigation of $\boldsymbol{F}$. verticillioides seed rot resistance.

(A ) Three disease grades were divided according to the infected area (IA, the area covered by mycelia on one seed / the total area of same seed). Grade I, $0<\mathrm{IA}[?] 25 \%$; Grade II, $25<\mathrm{IA}[?] 50 \%$; Grade III, $50<\mathrm{IA}[?] 100 \%$.

(B) The disease grades of seed rot were varied among indicated genotypes. Data are from a total of 150 seeds for each genotype.

Figure S6. Exogenous application of GA alters rice resistance toF. verticillioides.

(A ) Growth phenotype of KY131 rice seedlings. 14-d-old seedlings were sprayed with $\mathrm{H}_{2} \mathrm{O}$, GA (50 $\left.\mu \mathrm{M}\right)$ or uniconazole $(20 \mu \mathrm{M})$ once a day for 4 days, and then photographed. (B, C ) The disease symptoms of KY131 leaves upon F. verticillioides exposure. For B, 14-d-old seedlings were treated as panel (A), then sprayed with $F$. verticillioides spore suspension $(F . V)$ and sterile water (Mock) once a day for 6 days and 
photographed. For (C), 14-d-old seedlings were treated as panel (A), then the leaves were immersed in $F$. $V$ spore suspension for 5 days, then photographed.

Table S1. Primer sequences used in this study.

Hosted file

Figures.pdf available at https://authorea.com/users/456409/articles/553457-a-novel-maizemicrorna-negatively-regulates-the-resistance-to-fusarium-verticillioides 